\title{
Detection of nucleic acids with a novel stem-loop primer rolling circle amplification technique
}

\author{
Beibei Zhang ${ }^{1}$, Qiao Wang ${ }^{1}$, Jian Wu' ${ }^{1}$, Yin Chen², and Jinke Wang ${ }^{1 *}$ \\ ${ }^{1}$ State Key Laboratory of Bioelectronics, Southeast University, Nanjing 210096, China. ${ }^{2}$ School of Medical \\ Technology, Xuzhou Medical University, Xuzhou 221004, China
}

BioTechniques 64:69-80 (February 2018) doi 10.4155/btn-2017-0104

Keywords: stem-loop primer, rolling circle amplification, detecting, nucleic acid

This paper presents a new rolling circle amplification (RCA) technique using stem-loop primers (SLP). The technique enables detection of target DNA by either linear or exponential amplification (SLP-IRCA and SLPeRCA) in both liquid and solid phases. For solid-phase detection, SLP-eRCA detects nucleic acids in four steps: (1) covalently immobilize an array of capture probes (CP) on a solid support; (2) hybridize the CP array with the DNA sample; (3) incubate the CP array with an RCA reaction containing two SLPs; (4) image the CP array. SLP-eRCA detects nucleic acids in liquid phase in one step: a real-time RCA reaction containing the DNA sample and two SLPs. Both liquid- and solid-phase detection methods employ a general rolling circle and an SLP. The other SLP is specific to the target. The technique was verified by detecting synthesized oligonucleotides and six different human papillomaviruses (HPVs), both in liquid phase and on a solid surface. The technique also detected two high-risk HPVs (HPV16 and HPV18) in cervical carcinoma cells (HeLa and $\mathrm{SiHa}$ ) and clinical samples. This study provides proof-of-concept for the new RCA technique for nucleic acid detection, which overcomes major limitations of current RCA approaches.

Rolling circle amplification (RCA) has been widely used as an isothermal nucleic acid amplification technology $(1,2)$ that realizes target detection using circular templates and special DNA polymerases such as Phi29 (3). RCA can be divided into linear RCA (IRCA) and exponential RCA (eRCA). IRCA realizes amplification by elongating a single primer on a circular template, thus resulting in a low detection limit at the level of nM-pM $(4,5)$.

For improving RCA detection sensitivity $(6,7)$, various RCA methods have been developed, including exponential RCA (eRCA). The earliest eRCA method was developed using multiple primers for IRCA (3,8-10). However, the amplification efficiency of this method is often limited by the length of the circular template and the number of primers. Therefore researchers developed hyper-branched RCA (hbRCA), another ERCA method that relies on one or more primers complementary to the amplified products of IRCA $(3,8,11)$. $\mathrm{HbRCA}$ has remarkable sensitivity since it generates large numbers of copies of each circle in a short time $\left(10^{9}\right.$ or more copies in $90 \mathrm{~min}$ ) (8).

RCA detection can be realized both in solution and on solid supports. Liquidphase IRCA is often realized by annealing a specific padlock probe to its target, circularizing it with ligase, and polymerizing with a general primer complementary to the padlock probe. Liquid-phase eRCA is often realized by hbRCA $(3,8,11,12)$, multiple circles generated from the RCA products (13), and primer generation using nicking enzymes (10). However, it is difficult to realize homogeneous detection with the current liquid-phase RCA methods.

When RCA primers are directly or indirectly linked to a solid support, RCA products will be tethered to the solid support $(14,15)$, thus enabling the amplification signals to be localized (16). The major advantage of solid-phase RCA is that it enables simultaneous detection of multiple targets (17) and easy isolation of RCA products by briefly washing the solid support. However, the current solid-phase IRCA is limited by its low sensitivity (3-4 log on microarrays) and tedious padlock probe phosphorylation and ligation (18).

\section{METHOD SUMMARY}

To overcome the limitations of current rolling circle amplification (RCA) techniques and introduce an easy method for specifically and sensitively detecting target DNAs in liquid and solid phases, we developed a novel stem-loop primer RCA (SLP-RCA) technique that can be used to detect targets by both linear and exponential amplification (SLP-IRCA and SLP-eRCA). Solid-phase SLP-eRCA can detect targets with high sensitivity and throughput, while liquid-phase SLP-eRCA can detect targets with high sensitivity in a homogeneous format. 
Solid-phase eRCA can also be realized by performing hbRCA on a solid support, which improves sensitivity $(9-10$ log) (8). However, most of the amplified product is released into solution as free molecules (14), so the RCA products cannot be localized, which weakens the amplified signal for solid phase RCA and allows for interference among amplicons from different targets. Therefore, most solid-phase RCA detections rely on IRCA (3).

This study presents a new RCA technique using stem-loop primers (SLP) that can detect targeted nucleic acids in both IRCA and eRCA (SLP-IRCA and SLP-eRCA). For SLP-IRCA detection, only one SLP (SLP1) is used. The loop and stem sequence of SLP1 is specific to the target molecule and a general rolling circle $(\mathrm{RC})$. In the RCA reaction, the loop of SLP1 will hybridize with the target molecule, opening the stem and exposing the $3^{\prime}$ end, which will anneal with the RC. The $3^{\prime}$ end of SLP1 thus serves as a primer to start IRCA.

For SLP-eRCA detection, two SLPS (SLP1 and SLP2) are used. SLP1 is identical to that used in SLP-IRCA, while SLP2 is designed with a loop and stem sequence specific to the RCA product of SLP1 and the general RC, respectively. During the RCA reaction, SLP-IRCA is started by SLP1 and the loop of SLP2 hybridizes with the SLP-IRCA product. In this case, the stem of SLP2 is opened so that the exposed 3' end can anneal with the RC. The $3^{\prime}$ end of SLP2 thus serves as a primer and starts eRCA. The conformational change of SLP1 and SLP2 therefore triggers SLP-IRCA and SLP-eRCA, respectively.

This study demonstrates that both SLP-IRCA and SLP-eRCA can be used to detect target molecules such as chemically synthesized oligonucleotides and the L1 DNA fragments of various genotypes of human papillomaviruses (HPVs), both in liquid and solid phases.

\section{Materials and methods}

Preparation of SLP and

circular templates

All oligonucleotides used in this study are shown in Table 1. Each SLP was dissolved in a Tris- $\mathrm{Mg}^{2+}$ solution $(20 \mathrm{mM}$ Tris- $\mathrm{HCl}, \mathrm{pH}$ $8.0,3 \mathrm{mM} \mathrm{MgCl}_{2}$ ) at a concentration of 2.5 $\mu \mathrm{M}$. The SLP solution was incubated at $95^{\circ} \mathrm{C}$ for 5 min and then naturally cooled to room temperature. For preparing the circular templates, oligo $R C$ and $R C$ link were dissolved in TEN buffer, mixed, heated to $95^{\circ} \mathrm{C}$ for $5 \mathrm{~min}$, and slowly cooled to room temperature. The mixture was ligated overnight with T4 DNA ligase (Takara, Japan) at a final concentration of $14 \mathrm{U} / \mu \mathrm{L}$ at $16^{\circ} \mathrm{C}$. The reaction was heated at $65^{\circ} \mathrm{C}$ for $5 \mathrm{~min}$ and then treated overnight with exonuclease I (5 U/ML, Takara) and exonuclease

Table 1. Oligonucleotides used in this study.

Names

PC

NC

TO-CP

TO

SLP1

SLP2

mbSLP2

$\mathrm{RC}$

$\mathrm{RO}$

$\mathrm{RC}$ link

HPV6-CP

HPV11-CP

HPV16-CP

HPV18-CP

HPV33-CP

HPV35-CP

HPV6-spSLP1

HPV11-spSLP1

HPV16-spSLP1

HPV18-spSLP1

HPV33-spSLP1

HPV35-spSLP1

HPV6-IpSLP1

HPV11-IpSLP1

HPV16-IpSLP1

HPV18-IpSLP1

HPV33-IpSLP1

HPV35-IpSLP1

HPV16-E6-IpSLP1
Orientation, sequence and modification (Note: underlined bases refers to the annealing sequences as stem in SLP)

5'> $\mathrm{NH}_{2}$-TTTTTTTTTTTTTCATCATCACGCAGAGCATCATTT-Biotin $<3$ '

5'> $\mathrm{NH}_{2}$-TTTTTTTTTTTTATTCGGATCGCTCATCAGTTCTGC $<3$ '

5'>GCACTGACATCAAAGCAGCCAGGAGCAAACTCTTGGTACAGCTTTTTTTTTTT- $\mathrm{NH}_{2}<3$ '

5'>GTTGCTCTACCAATTGAGCTACACCGCTGTACCAAGAGTTTGCTCCTGGCTGCTTTGAT<3'

5'>GTATGTCTCCGGGTGTAGCTCAATTGGTAGAGCAACGGCAGACGGAGACATAC $<3$ '

5'>CCGTCTGCCTGTGTATCTTTGATTCGTCAGCCCTGTAAGGCAGACGG $<3$ '

5'>CTCCGTCTGCCT(6-FAM)GTGTATCTTTGATTCGTCAGCCCTGT(Dabcyl)AGGCAGACGGAG<3'

5'>P-CGTTATTTGTATTCTACTGTATTCTGTATGTCTCCGTCTGCCTGTCACCTGTGTATCTTTGATTCGTCAGCCCTGTATCC <3'

5' > TCCCGTTATTTGTATTCTACTGTATTCTTAGACC $<3$ '

5'>CAAATAACGGGATACAGGGC $<3$ '

5'>TATGTTAACACCCCAAGCGGTTTTTTTTTT- $\mathrm{NH}_{2}<3$ '

5'> GGGGGTAATAACAGATCATCTGTTTTTTTTTTT- $\mathrm{NH}_{2}<3$ '

5'>ATATCTACTTCAGAAACTACTTTTTTTTTTT- $\mathrm{NH}_{2}<3$ '

5'>CAGGTACAGGAGACTGTGTAGATTTTTTTTTTT- $\mathrm{NH}_{2}<3$ '

5'>CCTCCATCTGCTAGTTTACAGTTTTTTTTTTT- $\mathrm{NH}_{2}<3$ '

5'>GTAATGCTAACCAGGTAAAAGC TTTTTTTTTT- $\mathrm{NH}_{2}<3$ '

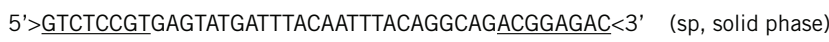

5'> GTCTCCGTGAGTTTGATTTACAGTTTACAGGCAGACGGAGAC $<3$ '

5'> GTCTCCGTGAATATGATTTACAGTITACAGGCAGACGGAGAC $<3$ '

5'> GTCTCCGTGAATATGATTTGCAGTTACAGGCAGACGGAGAC $<3$ '

5'> $\underline{\text { GTCTCCGTGAATATGATCTACAGTITACAGGCAGACGGAGAC }<3}$

5'> GTCTCCGTGAATATGATTACAGTTACAGGCAGACGGAGAC $<3$ '

5'> GTCTCCGTTATGTTAACACCCCAAGCGGAGGCAGACGGAGAC<3' (lp, liquid phase)

5'> GTCTCCGTGGGTAATAACAGATCATCTGAGGCAGACGGAGAC<3'

5'> GTCTCCGTATATCTACTTCAGAAACTACAGGCAGACGGAGAC<3'

5’> GTCTCCGTGGTACAGGAGACTGTGTAGAAGGCAGACGGAGAC $<3$ '

5'> GTCTCCGTCTCCATCTGCTAGTITACAGAGGCAGACGGAGAC $<3$ '

5'> GTCTCCGTAATGCTAACCAGGTAAAAGCAGGCAGACGGAGAC $<3$ '

5'> GTCTCCGTACTITAAACATTATCACATAGGCAGACGGAGAC $<3$ ' (targeting to E6 sequence) 
III (200 U/ $\mu \mathrm{L}$, Takara) at $37^{\circ} \mathrm{C}$. Finally, the reaction was inactivated at $80^{\circ} \mathrm{C}$ for $20 \mathrm{~min}$.

Preparation of capture probe array

The amino-modified oligonucleotides (Table 1) used as capture probes (CP) were dissolved in sterile water at a concentration of $10 \mu \mathrm{M}$. The oligonucleotides were diluted with 50\% dotting solution (CapitalBio, China) to a final concentration of $2 \mu \mathrm{M}$ and spotted on aldehyde-modified glass slides (CapitalBio) in a constant spotting volume of $35 \mathrm{~nL}$ per spot with a spotting robot (AD1500; BioDot, USA). The spotted slides were placed in a humidified chamber and incubated overnight at $37^{\circ} \mathrm{C}$. The slides were then washed with water for $2 \mathrm{~min}, 0.2 \%$ SDS for 2 min, and water for 2 min. Finally, the slides were kept in $0.15 \%$ (w/v) $\mathrm{NaBH}_{4}$ for 5 min and washed three times with water. The slides were then dried by spinning them in a slide centrifuge (Labnet, USA).

Detection of oligonucleotide with IpSLP-eRCA

Liquid-phase SLP-eRCA (IpSLP-eRCA) reactions were detected in two ways: electrophoresis and real time. For electrophoresis detection, the RCA reactions (10 $\mu \mathrm{L})$ consisted of $0.1 \mu \mathrm{M} R \mathrm{RC}$ template, $2 \times$ BSA (HYK, China), 0.5 mM dNTP, 1× phi29 DNA polymerase buffer ( $40 \mathrm{mM}$ Tris- $\mathrm{HCl}, 10$ $\mathrm{mM} \mathrm{MgCl}_{2}, 50 \mathrm{mM} \mathrm{KCl}, 5 \mathrm{mM}(\mathrm{NH} 4)_{2} \mathrm{SO}_{4}, 4$ mM DTT), 4 U phi29 DNA polymerase (HYK), 1.25 pmol SLP1, 1.25 pmol SLP2, and target oligonucleotide (TO), which were incubated at $30^{\circ} \mathrm{C}$ for $4 \mathrm{~h}$. The reaction was run on a $2 \%$ agarose gel. For real-time detection, the above RCA reactions $(10 \mu \mathrm{L})$ additionally included 1× SybrGreen I and $1.5 \mu \mathrm{M}$ report oligo $(\mathrm{RO})$. $\mathrm{RO}$ was used to anneal with the RCA product for SybrGreen I binding. The $R C A$ reaction was started at $30^{\circ} \mathrm{C}$ on a quantitative PCR (qPCR) machine (StepOne Plus; $\mathrm{ABI}$, USA) and the fluorescence signal was regularly collected at intervals of $30 \mathrm{~s}$ (regarded as 1 cycle).

Detection of oligonucle-

otide with spSLP-eRCA

For solid-phase SLP-eRCA (spSLP-eRCA) detection, the RC template $(0.1 \mu \mathrm{M})$, SLP1 $(0.1 \mu \mathrm{M})$ and $\mathrm{TO})$ were first mixed in $\mathrm{a} 50^{\circ} \mathrm{C}$ pre-heated hybridization solution $(12.5 \mu \mathrm{L})$ (15). The mixture was added to CP arrays. CP arrays were then covered with coverslips and incubated in a hybridization incubator (Robbins Scientific, USA) at $50^{\circ} \mathrm{C}$ for $1 \mathrm{~h}$. The slides with CP arrays were washed twice with sterile $\mathrm{H}_{2} \mathrm{O}$ for 2 min and spun dry. RCA reactions $(25 \mu \mathrm{L})$ containing $10 \mathrm{U}$ ф29 DNA polymerase (HYK), 1× $\phi 29$ buffer (HYK), 0.6 mM dNTP, 0.012 mM biotindUTP (Fermentas, USA), 0.1 $\mu \mathrm{M}$ SLP2, and $0.1 \mu \mathrm{M} R \mathrm{RC}$ were added to CP arrays. CP arrays were then covered with a coverslip and incubated in a humidified chamber (ArraylT, USA) at $30^{\circ} \mathrm{C}$ for $1 \mathrm{~h}$. The slide was washed twice with sterile $\mathrm{H}_{2} \mathrm{O}$ for 2 min. The slide was then detected with a common near infrared (NIRF) procedure as follows. The slide was successively incubated with a maleic acid buffer containing 1\% blocking reagents (Roche, Switzerland) and a maleic acid buffer containing 1:15000 diluted IRDye 800CW-Streptavidin (Licor, USA) at room temperature for $1 \mathrm{~h}$. Finally, the slides were washed twice in sterile $\mathrm{H}_{2} \mathrm{O}$ for 10 min and spun dry. The slides were imaged with a NIRF imager (Odyssey; Licor) with the channel of $800 \mathrm{~nm}$.

\section{Detection of HPV plasmid DNA with SLP-eRCA}

HPV plasmid harboring an HPV L1 fragment was denatured at $95^{\circ} \mathrm{C}$ for $10 \mathrm{~min}$ and chilled on ice. For detecting a particular HPV subtype with spSLP-eRCA, HPV plasmid, RC $(0.1 \mu \mathrm{M})$, and spSLP1 $(0.1 \mu \mathrm{M})$ were mixed in $50^{\circ} \mathrm{C}$ hybridization solution $(12.5 \mu \mathrm{L})$. The mixture was detected with CP arrays spotted with HPV CPs using the same procedure as for oligonucleotide detection. For detecting a particular HPV subtype with IpSLP-eRCA, the same RCA reactions $(10 \mu \mathrm{L})$ used for oligonucleotide detection were set up, replacing SLP1 and TO with IpSLP1 (Table 1) and denatured HPV plasmid DNA. The RCA reaction was detected using the same procedure as for oligonucleotide detection. In qualitative and quantitative detections, $0.5 \mathrm{pmol}$ and various amounts of HPV plasmid DNA were used, respectively.

\section{Detection of HPV DNA in ge-} nomic DNA with IpSLP-eRCA HepG2 genomic DNA (gDNA) was sheared by sonication and then denatured by heating as described above. For detecting a particular HPV subtype in a more complex DNA sample, the same RCA reactions (10 $\mu \mathrm{L}$ ) as above for IpSLP-eRCA detection were set up, in which 402 ng of HepG2 gDNA was added. The RCA reaction was detected with the same procedure as described above. For detecting HPV18 DNA in the gDNA of HeLa cells, the same RCA reactions ( $10 \mu \mathrm{L})$ as used in the previously described IpSLPeRCA detection were established, except that the HPV plasmid DNA was replaced with various amount of HeLa gDNA or 402 ng of HepG2 gDNA (as a control). The $\mathrm{RCA}$ reaction was detected with the same procedure as described above.

Detection of HPV DNA

with mbSLP-eRCA

For simplifying the IpSLP-eRCA reaction, SLP2 was modified into a molecular beacon (mbSLP2). For IpSLP-eRCA using mbSLP2 (mbSLP-eRCA), the signal was read using a qPCR machine (StepOne Plus; ABI). For qualitative detection of particular HPV subtypes, RCA reactions $(10 \mu \mathrm{L})$ contained $0.1 \mu \mathrm{M}$ RC template, $2 \times B S A, 0.5 \mathrm{mM}$ dNTP, $1 \times$ phi29 DNA polymerase buffer, 4 U phi29 DNA polymerase (HYK), 1.25 pmol IpSLP1, 1.25 pmol mbSLP2, and 0.5 pmol denatured HPV plasmid DNA. For quantitative detection of particular HPV subtypes, RCA reactions $(10 \mu \mathrm{L})$ contained $0.1 \mu \mathrm{M}$ RC template, 2x BSA, $0.5 \mathrm{mM}$ dNTP, 1× phi29 DNA polymerase buffer, $4 \cup$ phi29 DNA polymerase (HYK), 1.25 pmol IpSLP1, 1.25 pmol mbSLP2, various amounts of denatured particular HPV plasmid DNA or 208 ng of denatured HepG2 gDNA. For quantitative detection of HPV16 and HPV18 DNA in the gDNA of cervical carcinoma cells, the same RCA reaction was set up as for the HPV plasmid DNA quantitative detection, except that the HPV plasmid DNA was replaced with various amounts of denatured gDNA from cervical carcinoma cells including HeLa, SiHa, or C-33A. All RCA reactions were started at $10^{\circ} \mathrm{C}$ for 5 cycles, then $20^{\circ} \mathrm{C}$ for 5 cycles, followed by 140 cycles at $30^{\circ} \mathrm{C}$. The fluorescence intensity was regularly read with the qPCR machine at $30 \mathrm{~s}$ intervals. HeLa cells were used for HPV18 positive cervical carcinoma cells (19), SiHa cells for HPV16 positive cervical carcinoma cells (20), and C-33A cells for HPV-negative cervical carcinoma cell (21).

Detection of IpSLP-eRCA products with thermal denaturation The IpSLP-eRCA reactions (10 $\mu \mathrm{L})$ contained $0.1 \mu \mathrm{M}$ RC template, $2 \times$ BSA (HYK), 0.5 mM dNTP, 1×phi29 DNA polymerase buffer, 4 U phi29 DNA polymerase (HYK), 1.25 pmol SLP1, 1.25 pmol SLP2, 1×SybrGreen I, 1.5 $\mu \mathrm{M} \mathrm{RO}$, and $0.5 \mathrm{pmol}$ target oligonucleotide. The mbSLP-eRCA reactions $(10 \mu \mathrm{L})$ contained $0.1 \mu \mathrm{M}$ RC template, 2×BSA, $0.5 \mathrm{mM}$ dNTP, 1×phi29 DNA polymerase 
buffer, 4 U phi29 DNA polymerase (HYK, China), 1.25 pmol IpSLP1, 1.25 pmol mbSLP2, and 0.5 pmol TO. The negative reaction contained no TO. The fluorescence intensity of IpSLP-eRCA and mbSLPeRCA reactions was regularly read using a qPCR machine at 30 s intervals. When the $\mathrm{RCA}$ reaction finished, half of the reaction products were denatured at $95^{\circ} \mathrm{C}$ for 5 min and immediately chilled on ice. Then all denatured and undenatured reaction products were mixed with $10 \times$ loading buffer and run on a $2 \%$ agarose gel at 70 $\checkmark$ for $50 \mathrm{~min}$.

Detection of HPV DNA in clinical samples with mbSLP-eRCA

Cervical mucus exfoliated cells from eight patients at Jinling Hospital (Nanjing, China) were used as clinical samples. RCA reactions $(10 \mu \mathrm{L})$ contained $0.1 \mu \mathrm{M} R \mathrm{RC}$ template, $2 \times$ BSA, 0.5 mM dNTP, $1 \times$ phi29 DNA polymerase buffer, $4 \mathrm{U}$ phi29 DNA polymerase (HYK), 1.25 pmol IpSLP1, 1.25 pmol mbSLP2, 40 ng of various denatured DNAs that were from clinical samples. All
RCA reactions started at $10^{\circ} \mathrm{C}$ for 5 cycles, then $20^{\circ} \mathrm{C}$ for 5 cycles, followed by 140 cycles at $30^{\circ} \mathrm{C}$. The fluorescence intensity was read using a qPCR machine at $30 \mathrm{~s}$ intervals.

\section{Results and discussion \\ SLP-eRCA detection}

Detection of nucleic acid molecules with SLP-eRCA is schematically shown in Figure 1. SLP-eRCA detection in solid phase consists of four steps: (1) amino-modified oligonucleotides used as CPs are covalently immobilized on aldehyde-modified glass slides to create the CP array; (2) the CP array is hybridized with the DNA sample; (3) the CP array undergoes an $\mathrm{RCA}$ reaction containing SLP1, SLP2, and biotin-dUTP; (4) the CP array is incubated with $800 \mathrm{CW}$-streptavidin and imaged with a NIRF imager. SLP-eRCA detection in liquid phase is accomplished by performing an RCA reaction that contains the denatured DNA sample, SLP1, and SLP2.

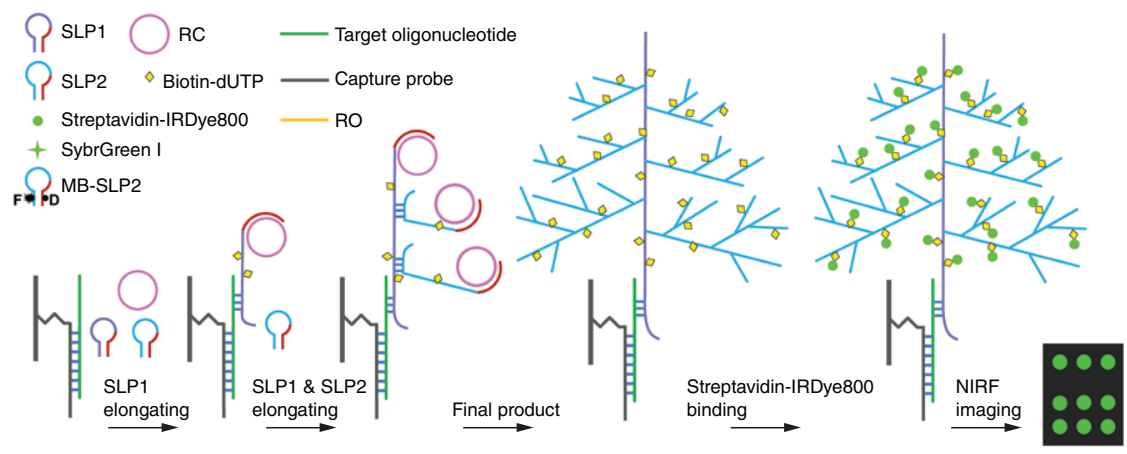

Solid-phase SLP-eRCA
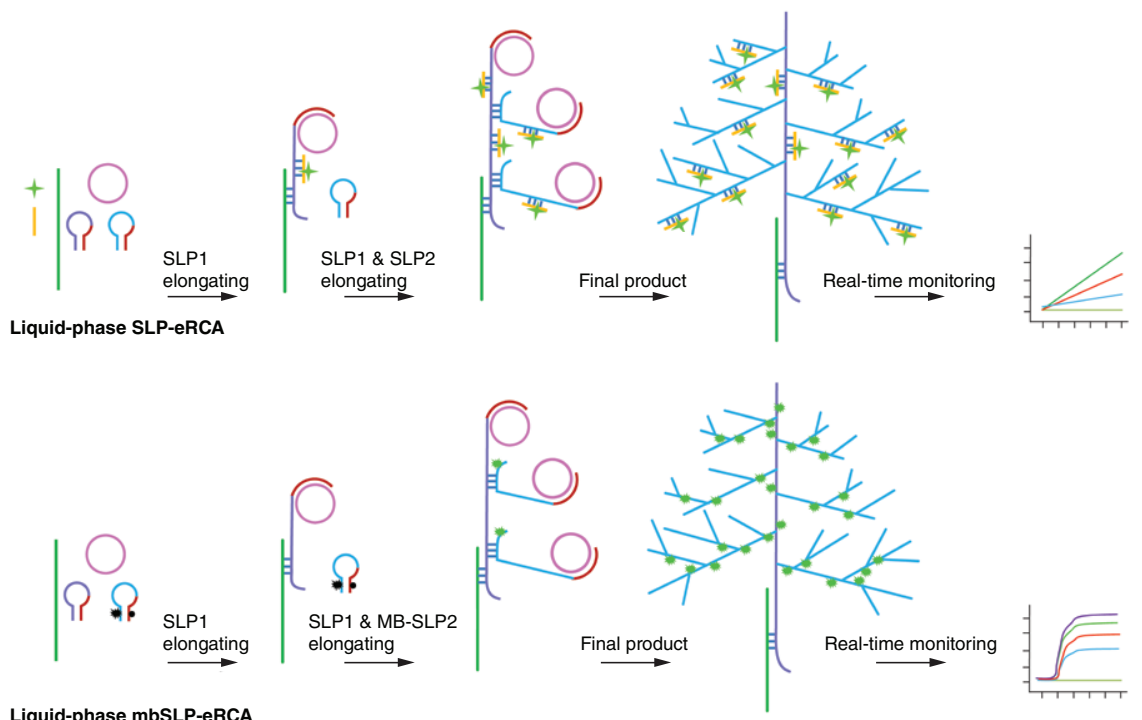

Detection of synthesized

DNA with IpSLP-eRCA

For exploring the feasibility of SLP-RCA, a synthesized TO was first detected with both SLP-IRCA and SLP-eRCA in liquid phase (IpSLP-IRCA and IpSLP-eRCA). Agarose gel electrophoresis revealed that both IpSLPIRCA and IpSLP-eRCA amplified products (Figure 2A). IpSLP-eRCA produced more amplified products than IpSLP-IRCA (Figure $2 A$ ), indicating that IpSLP-eRCA occurred in the presence of SLP2. The subsequent detection of various amount of TO demonstrated that IpSLP-eRCA could quantitatively detect TO (Figure 2B). Subsequently, including a reporter oligonucleotide $(\mathrm{RO})$ complementary to the RCA products and the dsDNA fluorescent dye SybrGreen I in the SLP-RCA reaction demonstrated that the signal readout of IpSLP-IRCA and IpSLP-eRCA reactions could be monitored in real-time with a gPCR machine (Figure 2C). IpSLP-eRCA showed a higher signal readout than IpSLP-IRCA in gel electrophoresis detection (Figure $2 \mathrm{C}$ ). Finally, it was also demonstrated that IpSLP-eRCA could quantitatively detect TO using the real-time signal readout (Figure 2D).

Detection of synthesized

DNA with spSLP-eRCA

Because solid-phase detection has advantages over liquid-phase detection, spSLP-RCA was next investigated. DNA arrays were first fabricated by coupling oligonucleotides used as a CP for the TO (TO-CP), a positive control (PC; modified with biotin), and a negative control (NC). Arrays were hybridized with varying amounts of TO. Arrays were then detected with an IRCA reaction (containing SLP1) or eRCA reaction (containing SLP1 and SLP2). As a result, TO was quantitatively detected by both spSLPIRCA (Figure 3A and 3B) and spSLP-eRCA (Figure $3 \mathrm{C}$ and $3 \mathrm{D}$ ). The detection limit of spSLP-eRCA (0.04 fmol) was higher than that of SPSLP-IRCA (1 fmol), indicating that spSLP-eRCA has a detection limit 25 fold more than spSLP-IRCA for the same reaction time (1 h). All PC spots showed similar signals in each array, indicating even coupling of probes on slides. The signal of $\mathrm{PC}$ spots in each array was used to normalize the signal of TO in the same array. For further improving the detection limit, the TO was detected again with spSLPeRCA by increasing the reaction time (4 h). The result indicated that the detection limit

Figure 1. Schematic illustration of the procedures for detecting nucleic acid molecules with SLP-eRCA. 
of SLP-eRCA significantly improved (Figure $3 \mathrm{E})$, with as little as 8 amol of TO detected.

\section{Detection of HPV DNA}

with spSLP-eRCA

We next investigated whether SLP-eRCA could detect HPV L1 DNA in more complex nucleic acid samples. DNA arrays fabricated with the capture probes for HPV16 and 18 were hybridized with HPV16 and 18 plasmid DNA. Arrays were then submitted to an RCA reaction containing SLP2 and spSLP1s for HPV16 and 18. The results revealed that $L 1$ DNA of the two HPV subtypes was specifically detected by spSLP-eRCA (Figure 4A).

Next, capture probes for an additional four HPV subtypes (HPV6, 11, 33, and 35) were designed and used to fabricate DNA arrays. Arrays were then hybridized with different HPV plasmids and submitted to an RCA reaction containing SLP2 and one HPV spSLP1 (Table 1). The results revealed that each HPV subtype was detected by spSLP-eRCA (Figure 4B and 4C).

Finally, we tested whether spSLP-eRCA could quantitatively detect HPV L1 DNA. DNA arrays fabricated with capture probes for HPV16 and 18 were hybridized with varying amounts of HPV16 and 18 plasmid DNA. Arrays were submitted to an RCA reaction containing SLP2 and spSLP1 for HPV16 and 18. The results revealed that two HPV subtypes were quantitatively detected by spSLP-eRCA (Figure 4D and 4E). Additionally, two HPV subtypes were detected with the same linear correlation $\left(R^{2}\right.$ $>0.9$ ) and a detection limit as low as $7.5 \mathrm{fmol}$.

\section{Detection of HPV DNA}

with IpSLP-eRCA

Next, whether HPV plasmid DNAs could be detected by IpSLP-eRCA was investigated. A set of 6 RCA reactions was prepared in which each reaction contained SLP2 and one HPV IpSLP1. The plasmid DNA of an individual HPV genotype was detected with each $\mathrm{RCA}$ reaction. The signal readout of $\mathrm{RCA}$ reactions was dynamically collected with a PCR machine.

The results revealed that $6 \mathrm{HPV}$ genotypes were detected by IpSLP-eRCA (Figure 5A). Additionally, further detection of HPV16 and 18 revealed that IpSLP-eRCA could quantitatively detect HPV DNAs (Figure 5B).

For exploring whether IpSLP-eRCA could detect HPV DNA in a more complex DNA sample, varying amounts of HPV16 or 18 plasmid DNA were mixed with 402 ng of HepG2 gDNA and detected with IpSLP-

eRCA. RCA was detected with SLP2 and IpSLP1 of HPV16 or 18. The results indicated that the two HPV DNAs mixed in gDNA were quantitatively detected by IpSLP-eRCA (Figure 5C).

Finally, for exploring whether IpSLPeRCA could detect HPV DNA integrated in the gDNA of cervical carcinoma cells, various amounts of HeLa gDNA were detected with IpSLP-eRCA using SLP2 and IpSLP1 for HPV18. The results revealed that HPV18 DNA integrated in HeLa gDNA was quantitatively detected by IpSLP-eRCA (Figure 5D).

\section{Detection of HPV DNA}

with mbSLP-eRCA

To simplify IpSLP-eRCA, we removed the reporter oligo $(\mathrm{RO})$ and SybrGreen I and

(A)

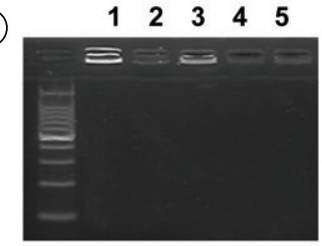

\begin{tabular}{lccccc}
\hline Reaction & $\mathbf{1}$ & $\mathbf{2}$ & $\mathbf{3}$ & $\mathbf{4}$ & $\mathbf{5}$ \\
\hline Target & + & - & + & - & + \\
SLP1 & + & + & + & + & - \\
SLP2 & + & + & - & - & + \\
\hline
\end{tabular}

(B)
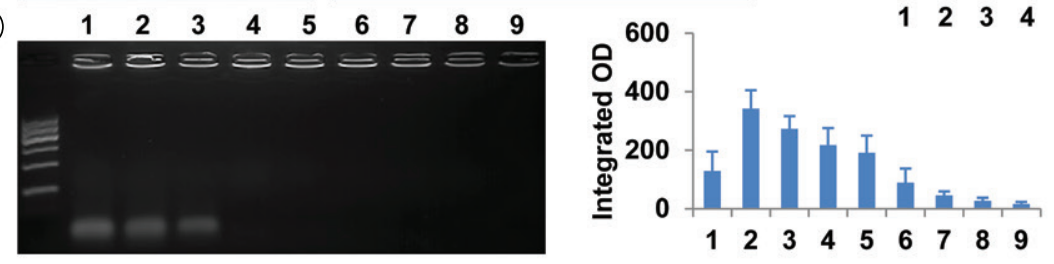

\begin{tabular}{llllllllll}
\hline Reaction & 1 & 2 & 3 & 4 & 5 & 6 & 7 & 8 & 9 \\
\hline Target (pmol) & 20 & 20 & 10 & 5 & 2.5 & 1.25 & 0.625 & 0.3125 & 0 \\
SLP1 (pmol) & 1.25 & 1.25 & 1.25 & 1.25 & 1.25 & 1.25 & 1.25 & 1.25 & 1.25 \\
SLP2 (pmol) & 0 & 1.25 & 1.25 & 1.25 & 1.25 & 1.25 & 1.25 & 1.25 & 1.25 \\
\hline
\end{tabular}

(C)

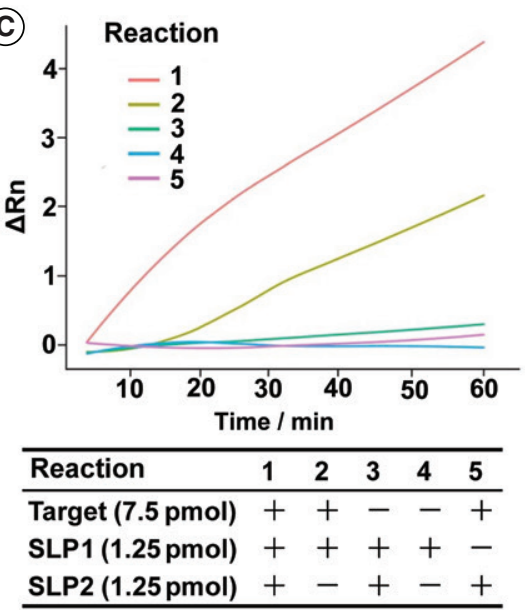

changed SLP2 into a molecular beacon by labeling two thymines in its sequence with FAM and Dabcyl (mbSLP2). This approach (mbSLP-eRCA) was successful for qualitative detection of various HPV subtypes and quantitative detection of HPV16 and 18 (Figure 6A and 6B).

Importantly, the detection limit of IpSLPeRCA greatly improved when using mbSLP2 (Figure 6B). As few as 5 fmol of HPV16 and 18 molecules were detected by mbSLPeRCA. Experiments with gDNA revealed that mbSLP-eRCA could also quantitatively and specifically detect HPV18 DNA integrated in HeLa gDNA and HPV16 DNA integrated in SiHa gDNA (Figure 7A and B). However, no HPV16 and HPV18 were detected in the gDNA of HepG2 cells (Figure 7A and B) or HPV-negative cervical carcinoma cells, 
(A)

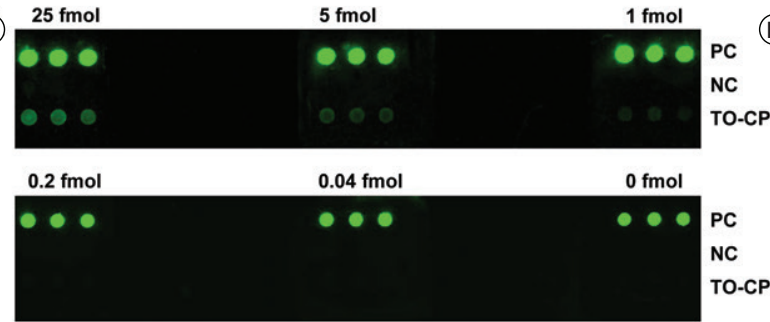

(C)

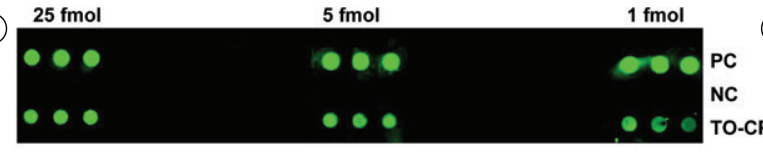

$0.2 \mathrm{fmol}$ $0.04 \mathrm{fmol}$
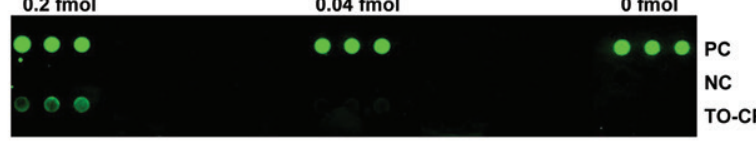

NC
TO-CP

E)

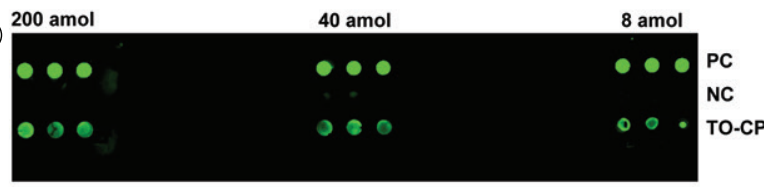

(B)

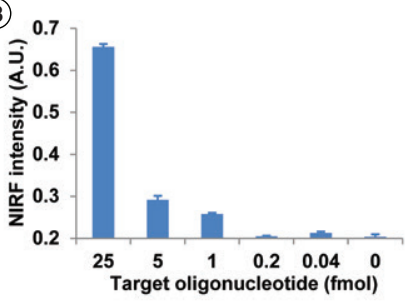

(D) 25

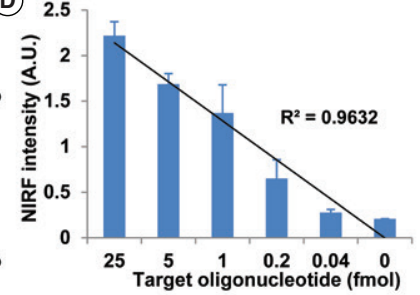

Figure 3. SpSLP-eRCA detection of a synthesized oligonucleotide. (A and B) Quantitative detection of a TO with spSLPIRCA running $1 \mathrm{~h}$. (C and D) Quantitative detection of TO with spSLP-eRCA running 1 h. (E) Quantitative detection of TO with spSLP-eRCA running $4 \mathrm{~h}$. The NIRF images ( $A, C$ and $E)$ and quantified signal intensity $(B$ and $D)$ of arrays are shown. Arrays were manufactured by spotting oligo TO-CP, PC, and NC on glass slides in triplicate. Arrays were hybridized with various amounts of TO $(25,5,1,0.2,0.04$, and 0 fmols) (A and C) or TO (200, 40, and $8 \mathrm{fmols}$ ) (E). Arrays then underwent a general spSLP-IRCA reaction containing SLP1 (A) or a general spSLP-eRCA reaction containing both SLP1 and SLP2 (C and $\mathrm{E})$.

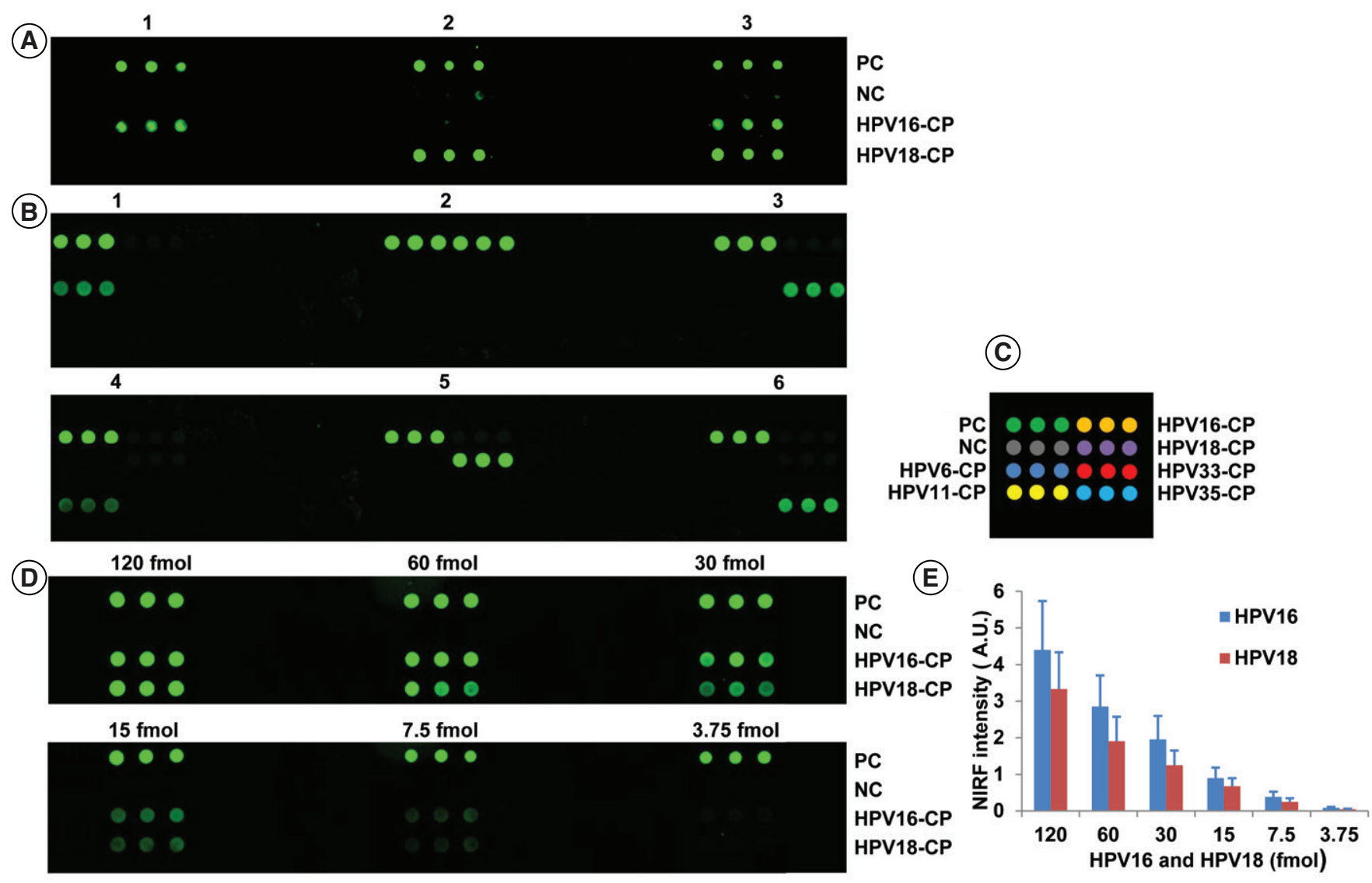

Figure 4. SpSLP-eRCA detection of HPV DNA. (A) Detection of HPV16 and HPV18 with spSLP-eRCA. Oligo CP, PC, and NC arrayed on glass slides in triplicate were hybridized with HPV16 (1), HPV18 (2), or HPV16\&HPV18 (3) DNA and detected with an RCA reaction containing HPV16-spSLP1, HPV18-spSLP1, and SLP2. (B and C) Detection of 6 different HPVs with spSLP-eRCA. The CPs of various HPVs, together with PC and NC, arrayed on glass slides in triplicate were hybridized with HPV6 (1), HPV16 (2), HPV33 (3), HPV11 (4), HPV18 (5), and HPV35 (6) DNA and detected with RCA reactions containing spSLP1 of 6 different HPVs and SLP2. (D and E) Quantitative detection of HPV16 and HPV18 with spSLP-eRCA. Oligo CPs, PC, and NC arrayed on glass slides in triplicate were hybridized with various mounts of HPV16 and HPV18 DNAs (120, 60, 30, 15, 7.5, and 3.75 fmols), and detected with an RCA reaction containing HPV16-spSLP1, HPV18-spSLP1, and SLP2. The NIRF images of the arrays (A, B, and D), layout of CPs in arrays (C), and quantified signal intensity of arrays (E) are shown. 

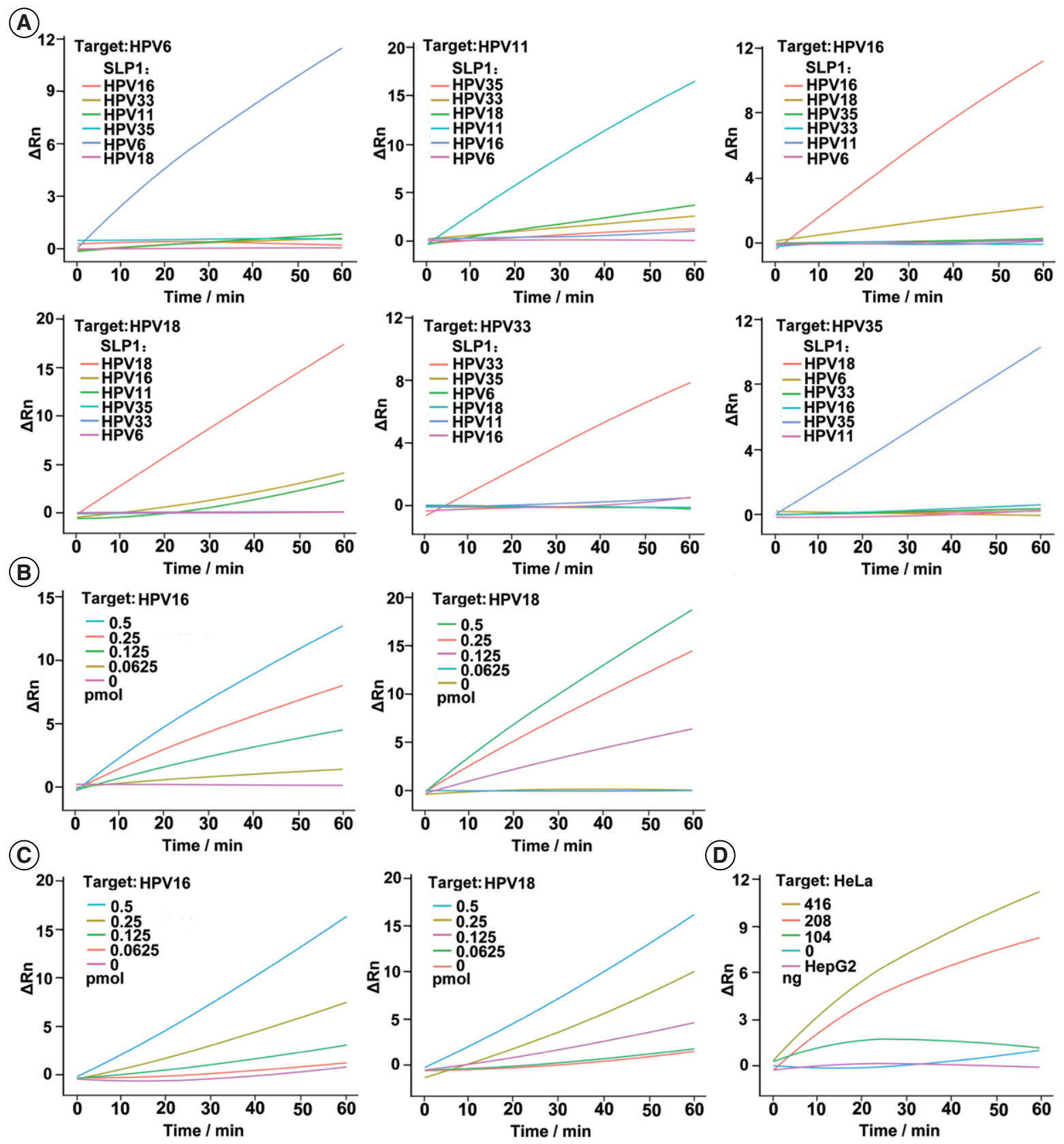

(D)

Figure 5. LPSLP-eRCA detection of HPV DNA. The IpSLP-eRCA reaction was monitored with a RT-PCR machine. (A) LPSLP-eRCA detection of 6 different HPV subtypes. A set of 6 RCA reactions containing SLP2 and one of HPVs-IpSLP1 was used to detect each subtype. The target HPV plasmid DNA was added to each of 6 reactions. (B) Quantitative detection of HPV16 and HPV18 plasmid DNA. The varying amounts of HPV plasmid DNA were detected with IpSLP-eRCA reactions containing SLP2 and HPV16-IpSLP1 or IpHPV18-SLP1. (C) Quantitative detection of HPV16 and HPV18 plasmid DNAs mixed in HepG2 gDNA. Varying amounts of HPV16 or HPV18 plasmid DNA were detected with the IpSLP-eRCA reactions containing HPV16-IpSLP1 or HPV18-IpSLP1 and SLP2. (D) Detection of HPV18 in HeLa cells. Varying amounts of HeLa gDNA were detected with IpSLP-eRCA reactions containing HPV18-IpSLP1 and SLP2. A SLPeRCA reaction only containing HepG2 gDNA (402 ng) was used as a negative control.

C-33A (Figure 7C). The results also demonstrated that the detection limit of IpSLP-eRCA was significantly improved by mbSLP-eRCA.

\section{Detection of IpSLP-eRCA prod-}

ucts with thermal denaturation

For exploring whether a tree-like complex was formed during SLP-eRCA reactions, a new detection of IpSLP-eRCA and mbSLPeRCA products was performed. Oligonucle- otide TO was simultaneously detected with IpSLP-eRCA and mbSLP-eRCA (Figure 8A and $C)$. Then, the IpSLP-eRCA and mbSLPeRCA reactions were divided, with one half denatured by heating and chilling on ice. All denatured and undenatured reactions were then loaded on an agarose gel for electrophoresis. The results revealed that smears appeared in the lanes loaded with the denatured reactions but not those loaded with the undenatured reactions (Figure 8B and D), indicating that exponential amplification truly occurred and a tree-like complex was formed in IpSLP-eRCA and mbSLPeRCA reaction.

Detection of HPV DNA in clinical samples with mbSLP-eRCA Finally, HPV genes in eight clinical samples (cervical mucus exfoliated cells) were 

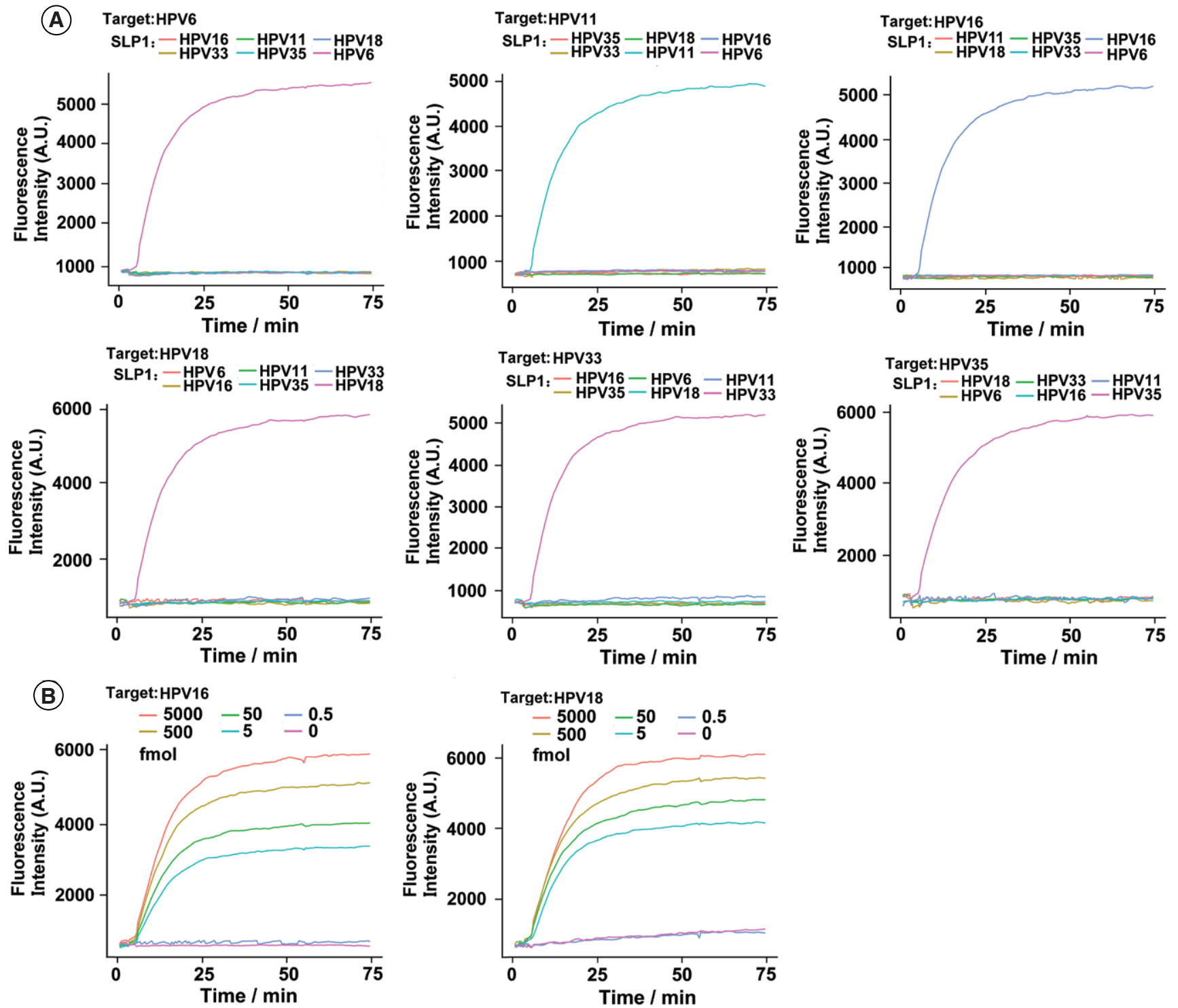

Figure 6. MbSLP-eRCA detection of HPV DNA cloned in plasmids. (A) Detection of 6 different HPV subtypes with mbSLP-eRCA. In detecting each subtype, 6 RCA reactions containing mbSLP2 and one of HPVs-IpSLP1 were used. The target HPV plasmid DNA was added to each of the reactions. (B) Quantitative detection of HPV16 and HPV18 plasmid DNA mixed in HepG2 gDNA. HepG2 gDNA was included in each reaction (208 ng). Varying amounts of HPV16 or HPV18 plasmid DNA were detected with mbSLP-eRCA reactions containing HPV16-IpSLP1 or HPV18-IpSLP1 and mbSLP2.

detected using mbSLP-eRCA. The results revealed that HPV16 was present in two clinical samples (samples 1 and 2), and HPV18 existed in one clinical sample (sample 3) (Figure 9). These results agree with the HC2 (Digene) test reports from Jinling Hospital, suggesting the reliability of mbSLP-eRCA detection.

Applications for SLP-RCA

$\mathrm{HPV}$ is a dsDNA virus that causes cervical, anal, and other cancers (22). There are around 100 types of HPV with different genetic variations (23). With respect to oncogenic potential, HPVs are divided into high-risk HPV (hrHPV) and low-risk HPV (IrHPV) (24). The most common worldwide hrHPVs are
HPV-16 and 18 (25-27), which lead to approximately $70 \%$ of cervical cancers (28). Other hrHPVs include 31, 33, 35, $39,45,51,52,56,58,59,68,82$, and others (29). IrHPVs include 6, 11, 40, 42, $43,44,61,81$, and others $(30,31)$. At present, HPV clinical detection is widely performed for cervical cancer diagnosis and routine health examinations using various PCR-based techniques $(27,28)$.

This study employs HPV DNA as a nucleic acid target for SLP-RCA detection. The results indicate that SLP-RCA can detect HPV DNAs both in liquid and solid phase. It was found that spSLP-eRCA had a detection limit of 8 amol. LpSLP-eRCA detected
HPV DNA with a detection limit of 5 fmol. MbSLP-eRCA detected HPV16 and HPV18 DNA in as little as $26 \mathrm{ng}$ of gDNA from two cervical carcinoma cell lines. However, this study focused on validating the SLP-RCA method by using HPV as a convenient experimental material; the purpose was not to develop a new HPV detection kit for clinical application.

These data demonstrate that RCA can be accomplished using SLP. To our knowledge, this is the first report of RCA with such a method. SLP-RCA has advantages over currently used IpRCA, which relies on synthesizing a phosphorylated padlock probe and ligating it with DNA 

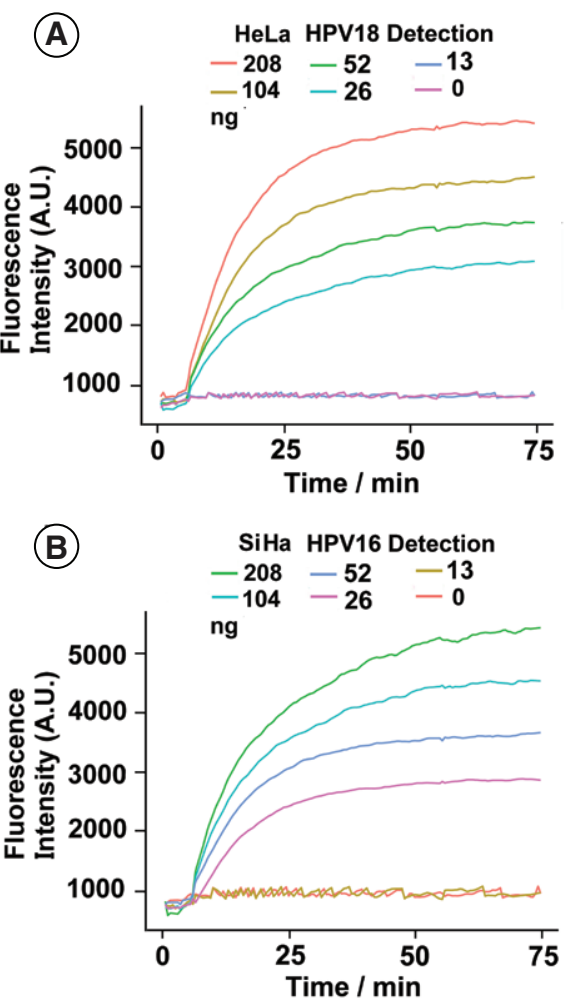
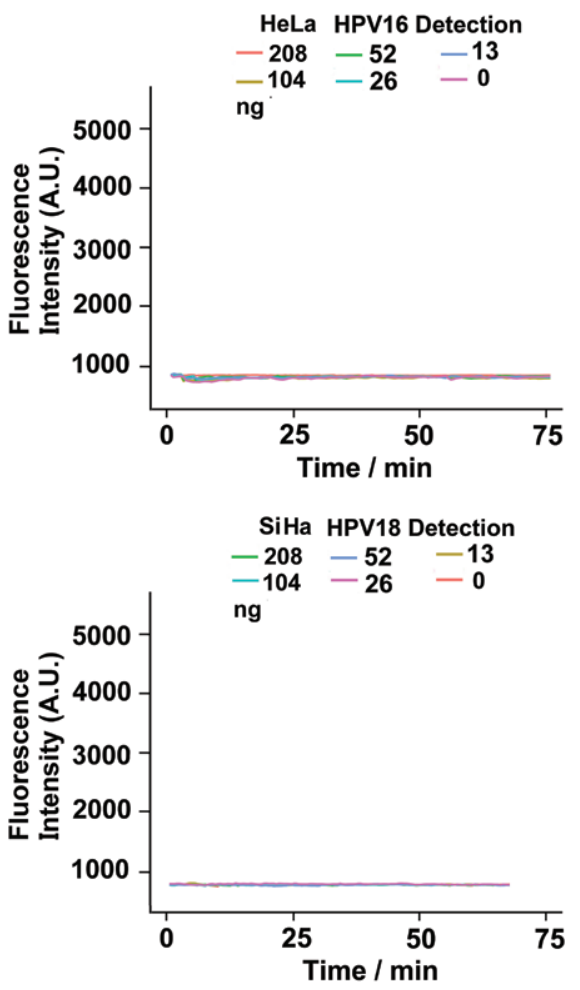

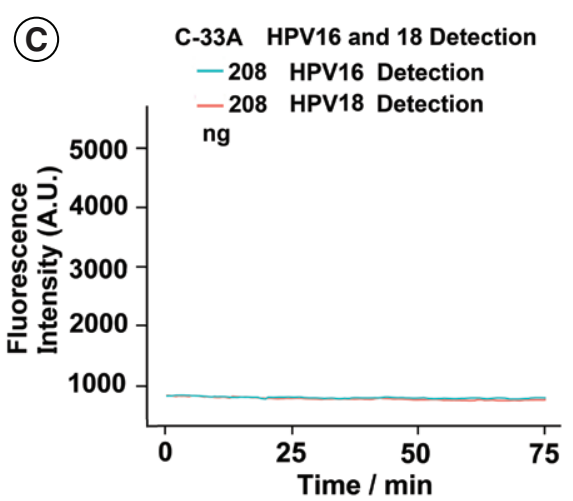

Figure 7. MbSLP-eRCA detection of HPV DNA in gDNA of cervical carcinoma cells. (A and B) Detection of HPV18 L1 or HPV16 E6 in HeLa and SiHa cells. Varying amounts of HeLa or SiHa gDNA were detected with mbSLP-eRCA reactions containing HPV18-IpSLP1 or HPV16-E6-IpSLP1 and mbSLP2. An SLPeRCA reaction containing only HepG2 gDNA was used as a negative control. (C) Detection of HPV18 L1 and HPV16 E6 in C-33A cells.

(A)

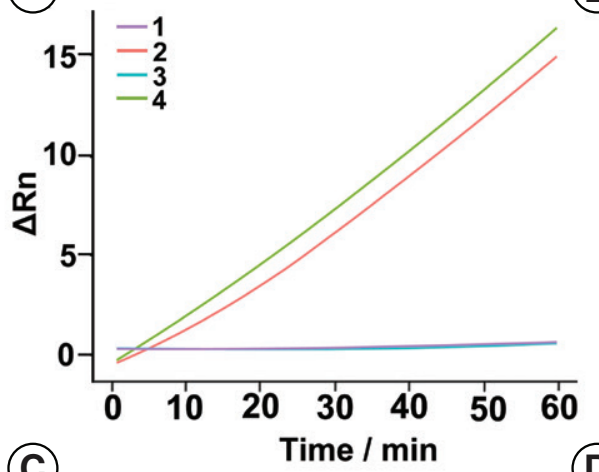

(C)

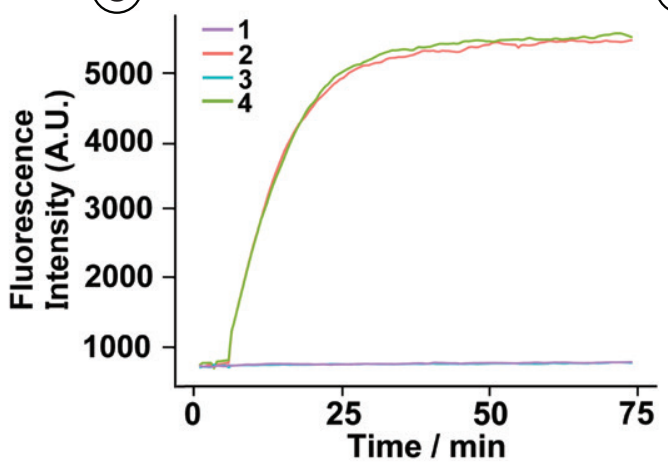

(B)

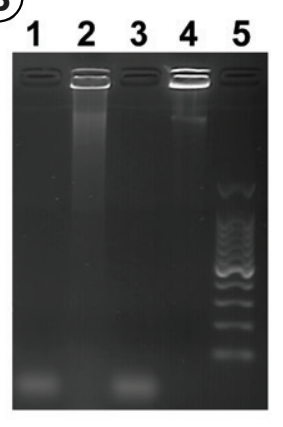

(D) $\begin{array}{lllll}1 & 2 & 3 & 4 & 5\end{array}$

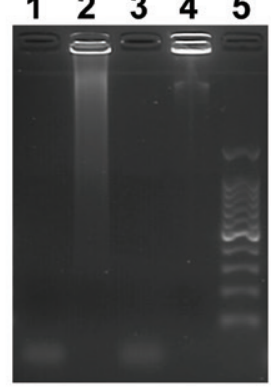

Figure 8. Detection of eRCA products with thermal denaturation. (A) Fluorescence quantitation results from IpSLP-eRCA. (B) Electrophoretic results of IpSLP-eRCA. 1, NC (thermally denatured); 2, IpSLP-eRCA (thermally denatured); 3, NC; 4, IpSLP-eRCA; 5, 100bp DNA ladder. (C) Fluorescence quantitation results from mbSLP-eRCA. (D) Electrophoretic results from mbSLP-eRCA. 1, NC (thermally denatured); 2, mbSLP-eRCA (thermally denatured); 3, NC; 4, mbSLP-eRCA; 5, 100bp DNA ladder. 

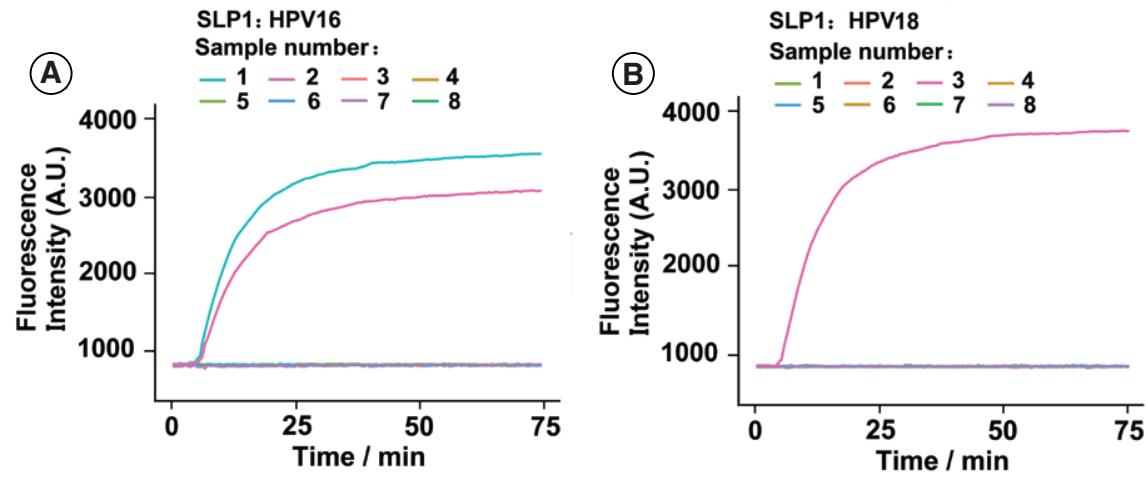

Figure 9. MbSLP-eRCA detection of HPV DNA in eight clinical samples. (A) Detection of HPV16 in eight clinical samples. Sample 1 and 2 were HPV16 positive. (B) Detection of HPV18 in eight clinical samples. Sample 3 was HPV18 positive.

ligase for detection $(32,33)$. SLP-RCA employs a general circular template, which eliminates the DNA hybridization and ligation steps needed for the current IpRCA approach, thus enabling real-time homogeneous detection at a constant temperature $\left(30^{\circ} \mathrm{C}\right)$. The homogeneous detection is helpful for technical applications. By using some visible signal readout methods such as hydroxylnaphthol blue (HNB) (34), SybrGreen (34), nanoparticles $(1,32,35)$, or DNA enzyme-catalyzed colorimetric reactions (36), SLP-RCA has the potential for use in field detection.

SpSLP-eRCA has a unique advantage over currently used eRCA techniques. In spSLP-eRCA detection, all RCA products are linked together as a highly branched complex immobilized on a solid support, which prevents RCA products from releasing into solution as free molecules. In this study, we detected the SLP-eRCA products with agarose gel electrophoresis and found no free DNA in the lane. However, thermal denaturation of IpSLP-eRCA and mbSLP-eRCA products produced free DNAs that showed up as smears after agarose gel electrophoresis. These results indicate that no free DNA was released from the highly branched complex produced by SLP-eRCA reaction. Gel electrophoresis of hbRCA, however, appears as a DNA ladder in the lane (8), indicating that many RCA products were displaced into solution as free DNA molecules in the hbRCA reaction. The free DNA released during RCA may result in low detection sensitivity and interference among amplif- icons from different targets. Additionally, the current method for hbRCA relies on multiple primers for hyper branch amplification; however, SLP-eRCA uses only one molecular switch primer (SLP2) for exponential amplification. Therefore, SLP-eRCA is suitable for detection on solid supports, such as glass slides (15), porous substrates (16), or beads $(1,35,37)$.

The SLP-eRCA technique developed here is adaptable. In this study, SybrGreen and IRDye800CW were used as signal readouts for SLP-eRCA. SLP-eRCA can also be reported with molecular beacons (38-40). In fact, the SLP2 used in this study can be easily modified to become a molecular beacon by labeling it with a donor and acceptor pair for fluorescence resonance energy transfer (FRET). In this case, SLP-eRCA can be directly reported using SLP2 itself. Additionally, mbSLP2 can be used to report SLP-eRCA both in liquid and solid phases. To verify the applicability of mbSLP2 in SLP-eRCA, we performed mbSLP-eRCA detection. The results revealed that mbSLP2 not only faciliatated the qualitative and quantitative detections of target molecules, but also improved the detection limit of IpSLP-eRCA. In fact, other fluorescent signal readouts can also be used for SLP-eRCA. For example, fluorescently labeled mononucleotides such as Cy3-dCTP can be used in spSLP-eRCA, which can be detected with currently available DNA microarray scanners (14). Additionally, the capture probe can be removed from spSLP-eRCA detection by directly using immobilized SLP1
$(41,42)$. In this case, hybridization of target molecules with the immobilized SLP1 will directly start the spSLP-eRCA reaction.

Finally, it is important to note that the stable stem-loop structure is critical for SLP-eRCA. Therefore, some optimization experiments in SLP design were performed prior to this study (data not shown). For example, stems with different lengths were explored, and the best was then employed in this study. An optimized general stem sequence was applied to all SLP1s (Table 1). A target sequence compatible with the optimized general stem sequence was selected when designing this study. The specific hybridization between SLP1 and target nucleic acids is critical for SLP-eRCA detection.

\section{Acknowledgments}

This work was supported by grants from the National Natural Science Foundation of China (Grant 61571119 and 81702103) and the Jiangsu Provincial Natural Science Foundation (No.BK20170252). The authors declare no competing financial interests.

\section{References}

1. Zhao, W., M.M. Ali, M.A. Brook, and Y. Li. 2008. Rolling circle amplification: applications in nanotechnology and biodetection with functional nucleic acids. Angewandte Chemie International Edition 47:6330-6337.

2. Schweitzer, B., S. Roberts, B. Grimwade, W. Shao, M. Wang, Q. Fu, Q. Shu, I Laroche, et al. 2002. Multiplexed protein profiling on microarrays by rolling-circle amplification. Nat. Biotechnol. 20:359-365.

3. Ali, M.M., F. Li, Z. Zhang, K. Zhang, D.-K. Kang, J.A. Ankrum, X.C. Le, and W. Zhao. 2014. Rolling circle amplification: a versatile tool for chemical biology, materials science and medicine. Chem. Soc. Rev. 43:3324-3341.

4. Wu, Z.-S., H. Zhou, S. Zhang, G. Shen, and R. Yu. 2010. Electrochemical aptameric recognition system for a sensitive protein assay based on specific target binding-induced rolling circle amplification. Anal. Chem. 82:2282-2289.

5. Zhu, X., H. Xu, H. Zheng, G. Yang, Z. Lin, B. Qiu, L. Guo, Y. Chi, and G. Chen. 2013 An ultrasensitive aptameric sensor for proteins based on hyperbranched rolling circle amplification. Chem. Commun. 49:10115-10117.

6. Thomas, D.C., G.A. Nardone, and S.K. Randall. 1999. Amplification of padlock probes for DNA diagnostics by cascade rolling circle amplification or the polymerase chain reaction. Arch. Pathol. Lab. Med. 123:11701176. 
mental and Therapeutic Medicine 10:21692174.

27. Rao, A., S. Young, H. Erlich, S. Boyle, M. Krevolin, R. Sun, R. Apple, and C. Behrens. 2013. Development and characterization of the cobas human papillomavirus Test. J. Clin. Microbiol. 51:1478-1484.

28. Abreu, A.L., R.P. Souza, F. Gimenes, and M.E. Consolaro. 2012. A review of methods for detect human Papillomavirus infection. Virol. J. 9:262.

29. Doorbar, J., W. Quint, L. Banks, I.G. Bravo, M. Stoler, T.R. Broker, and M.A. Stanley. 2012. The biology and life-cycle of human papillomaviruses. Vaccine 30:F55-F70.

30. Tao, P., W. Zheng, Y. Wang, and M.-I. Bian. 2012. Sensitive HPV genotyping based on the flow-through hybridization and gene chip. BioMed Research International 2012.

31. Syrjänen, S., G. Lodi, I. von Bültzingslöwen, A. Aliko, P. Arduino, G. Campisi, S. Challacombe, G. Ficarra, et al. 2011. Human papillomaviruses in oral carcinoma and oral potentially malignant disorders: a systematic review. Oral Dis. 17:58-72.

32. Lin, C., Y. Zhang, X. Zhou, B. Yao, and Q. Fang. 2013. Naked-eye detection of nucleic acids through rolling circle amplification and magnetic particle mediated aggregation. Biosens. Bioelectron. 47:515-519.

33. Li, J.J., Y. Chu, B.Y. Lee, and X.S. Xie. 2008 Enzymatic signal amplification of molecular beacons for sensitive DNA detection. Nucleic Acids Res. 36:e36.

34. Hamidi, S.V., H. Ghourchian, and G. Tavoosidana. 2015. Real-time detection of H5N1 influenza virus through hyperbranched rolling circle amplification. Analyst 140:15021509.

35. Xing, Y., P. Wang, Y. Zang, Y. Ge, Q. Jin, J. Zhao, X. Xu, G. Zhao, and H. Mao. 2013. A colorimetric method for H1N1 DNA detection using rolling circle amplification. Analyst 138:3457-3462.

36. Tang, L., Y. Liu, M.M. Ali, D.K. Kang, W. Zhao, and J. Li. 2012. Colorimetric and ultrasensitive bioassay based on a dual-amplification system using aptamer and DNAzyme. Anal. Chem. 84:4711-4717.

37. Li, Y., Y. Zeng, X.T. Ji, X. Li, and R. Ren. 2012. Cascade signal amplification for sensitive detection of cancer cell based on self-assembly of DNA scaffold and rolling circle amplification. Sensor Actuat B-Chemical 171:361-366.
38. Tyagi, S. and F.R. Kramer. 1996. Molecular beacons: probes that fluoresce upon hybridization. Nat. Biotechnol. 14:303-308.

39. Tyagi, S., D.P. Bratu, and F.R. Kramer. 1998. Multicolor molecular beacons for allele discrimination. Nat. Biotechnol. 16:49-53.

40. Li, J.J., R. Geyer, and W. Tan. 2000. Using molecular beacons as a sensitive fluorescence assay for enzymatic cleavage of singlestranded DNA. Nucleic Acids Res. 28:e52-e52.

41. Wang, H., J. Li, H. Liu, Q. Liu, Q. Mei, Y. Wang, J. Zhu, N. He, and Z. Lu. 2002. Labelfree hybridization detection of a single nucleotide mismatch by immobilization of molecular beacons on an agarose film. Nucleic Acids Res. 30:e61.

42. Wang, D.C., L.H. Hu, H.M. Zhou, E.S. AbdelHalim, and J.J. Zhu. 2013. Molecular beacon structure mediated rolling circle amplification for ultrasensitive electrochemical detection of microRNA based on quantum dots tagging. Electrochem. Commun. 33:80-83.

First draft submitted: 1 November 2017; Accepted for publication: 19 January 2018.

Address correspondence to Jinke Wang, State Key Laboratory of Bioelectronics, Southeast University, Nanjing 210096, China. Tel.: +86 25 83793620; fax: +86 25 83793620; E-mail: wangjinke@seu.edu.cn

To purchase reprints of this article contact: s.cavana@future-science.com

\section{LOSE THE WOBBLE! LOSE THE DRIP!}

Unique, innovative, patented 2-tier step design: - Less Force needed to insert - $2 X$ contact in your controller's nozzle - Less Jamming AND Less Wobble! - Less Wobble means Less Dripping! - Works with ANY pipet controller

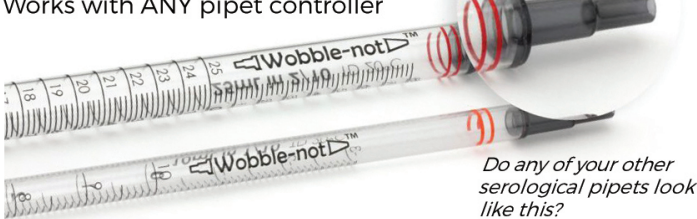
like this? $=\pi$ Wobble-not $D^{\text {TM }}$

The first-ever ergonomic Serological Pipet

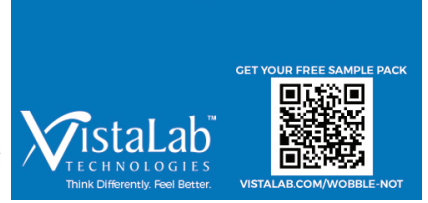

